\title{
An evolutionary perspective on the development of primate sociality
}

\author{
Federica Amici $^{1,2,3} \cdot$ Anja Widdig ${ }^{1,2}$
}

Received: 29 June 2019 / Accepted: 5 July 2019 /Published online: 1 August 2019

(C) Springer-Verlag GmbH Germany, part of Springer Nature 2019

\begin{abstract}
It is now well established that sociality plays a crucial role in primates. For example, among non-human primates, individuals with strong social bonds significantly increase their fecundity, reproductive success, offspring survival and longevity. Also in humans, social integration positively affects psychological stress, health and survival. However, despite the evidence that sociality is a fundamental fitness trait, relatively little is known about how sociality develops. Previous studies are mainly limited to the development of human sociality in Western cultures. Hence, to increase our knowledge about important human developmental milestones, an inter-specific and inter-cultural approach is needed. In particular, to understand the evolutionary roots of human sociality, studies on non-human primates are of large importance. The present Topical Collection is divided into four parts addressing (i) the effect of main bonding partners (mothers, siblings, peers) on social development in non-human primates; (ii) other factors affecting social development, such as sex differences, genetic constraints and personality; (iii) how social cognition and multimodal communication develop through early life in non-human primates and (iv) how human sociality develops through ontogeny, with a special focus on cross-cultural differences. In this paper, we introduce the development of sociality from an evolutionary perspective, briefly summarise each contribution of this Topical Collection and close with possible future directions. We aim to summarise the current state of research on the development of primate sociality, stimulate inter-disciplinary research and provide insight into the outstanding variability in social development across non-human primate species and human cultures.
\end{abstract}

\section{Significance statement}

This paper opens a Topical Collection on the development of primate sociality. Here, we introduce the topic, summarise contributions to this collection and outline future directions. To understand the evolutionary roots of human sociality, we consider the social development across different non-human primate species and human cultures.

Keywords Development of primate sociality $\cdot$ Ontogeny $\cdot$ Non-human primates $\cdot$ Human cultures $\cdot$ Social evolution

Communicated by T. Bakker

This article is a contribution to the Topical Collection An evolutionary perspective on the development of primate sociality - Guest Editors: Federica Amici and Anja Widdig

Anja Widdig

anja.widdig@eva.mpg.de

1 Research Group of 'Primate Kin Selection', Department of Primatology, Max-Planck Institute for Evolutionary Anthropology, Deutscher Platz 6, 04103 Leipzig, Germany

2 Institute of Biology, Faculty of Life Science, University of Leipzig, Talstraße 33, 04103 Leipzig, Germany

3 Leipzig Research Center for Early Child Development, University of Leipzig, Leipzig, Germany

\section{Introduction}

Sociality, or the tendency to associate and interact with other group members, plays a crucial role in primate lives. In nonhuman primates, strong and enduring social bonds (i.e. preferential affiliative relationships) significantly increase individual fitness in terms of fecundity, reproductive success, offspring survival and longevity (e.g. Silk et al. 2003, 2009, 2010; Schülke et al. 2010; Kulik et al. 2012; Archie et al. 2014; but see Thompson and Cords 2018). Also in humans, social integration positively affects psychological stress, health and survival (Smith and Christakis 2008; Holt-Lunstad et al. 2010).

Despite the fact that sociality is a fundamental fitness trait, we still understand little about how sociality develops. There are at least two reasons for that. Firstly, most of our knowledge is limited to the development of human sociality, with only few studies having systematically addressed how sociality develops 
during ontogeny in non-human primates (e.g. Lonsdorf et al. 2014; Kulik et al. 2015a, 2015b) or which factors during early development affect social connectedness later in life (e.g. Tung et al. 2016). As a consequence, we understand little about the evolutionary roots of human sociality. Specifically, we have little knowledge about which influencing factors or circumstances experienced during early development affect sociality and hence individual survival and fitness. Given that these are factors that can be easier to access in non-human primate groups, a comparative perspective is likely to be very informative for those interested in human mental and physical health and survival. Secondly, despite the existence of important cross-cultural differences on human sociality, researchers have only recently started investigating differences in developmental trajectories across cultures other than Western ones (see for example Henrich et al. 2010; Nielsen and Haun 2015). This lack of inter-specific and inter-cultural studies is especially problematic, because it does not allow drawing reliable conclusions on human developmental milestones, and hence makes extrapolation to other species also problematic. The inclusion of non-human primates would be especially important, because studies on natural populations not only have the advantage of enhancing the understanding of our evolutionary past but also provide more objective measurements of sociality in comparison with most human studies, which are often restricted to self-assessments using questionnaires. Hence, studies on the development of sociality in non-human primates can significantly contribute to our understanding about the link between sociality and fitness.

In this Topical Collection, we bring together leading experts on different developmental aspects of sociality, across several primate species and human cultures, in order to discuss recent advances in the study of social development in human and non-human primates, and point to future directions for research. With this Topical Collection, we have three main aims. Firstly, we want to serve as a reference for the current state of research on the development of primate sociality from an evolutionary perspective, by including cross-cultural and inter-specific studies. Secondly, we aim to stimulate future research on the topic, with a special focus on under-studied research areas. Thirdly, we want to promote inter-disciplinary dialogue and future collaboration, in order to gain a more comprehensive understanding of primate social development and its evolutionary origins. Furthermore, we hope to highlight the importance of primate social development, and its long-term effects on fitness and survival, but also provide an insight into the outstanding variability in social development across primate species and human cultures.

\section{State of the art}

It has long been established that maternally related individuals of different non-human primate species form strong social relationships with each other (reviewed in Chapais 2001; Silk 2002a, Silk et al. 2009). However, even unrelated individuals are known to often engage in long-term relationships with each other (for example Silk 2002b; Seyfarth and Cheney 2012). In evolutionary terms, long-lasting social bonds among conspecifics allow individuals to significantly increase their fitness, independently of relatedness (Silk et al. 2003, 2009, 2010; Schülke et al. 2010; Archie et al. 2014).

Several proximate mechanisms likely mediate the correlation between social bonds and fitness, even among unrelated partners (Silk 2014). Non-human primates, for instance, have been shown to gain direct fitness benefits from bonding with other group members. For example, by obtaining more coalitionary support from bonding partners, males either ascend in dominance or more successfully sire offspring (Schülke et al. 2010; Gilby et al. 2013). Social bonds can provide further direct fitness benefits by resulting in efficient protection against both predators' (Michelleta et al. 2012) and conspecifics' harassment (Moscovice et al. 2009; Nguyen et al. 2009), or by securing greater access to food resources (King et al. 2012; Haunhorst et al. 2017). Moreover, non-human primates have been reported to also gain indirect benefits by forming strong social bonds. Prolonged activation of the stress system, in particular, can have long-term negative effects on primate health and reproduction (Wingfield and Sapolsky 2003). By reducing stressful situations like social isolation, social instability and subordinate status, social bonds may thus have a significant positive effect on stress response systems in primates, on their immune function and ultimately on their health and survival (see Silk 2014). Indeed, strong social relationships have been shown to have a positive effect on the ability to deal with stressful events (for females: Engh et al. 2006a, 2006b; Crockford et al. 2008; Wittig et al. 2008; for both sexes: Wittig et al. 2016). Furthermore, strong bonds among females have a positive effect on their longevity (Silk et al. 2010) and on their infant survival (Silk et al. 2003, 2009).

Although social behaviour has a strong genetic component (reviewed in Ebstein et al. 2010), it is also importantly affected by the socio-ecological conditions experienced throughout life. Human children, for instance, slowly acquire the social competences, which are necessary to interact with others, and gradually grow into the social roles characterising them as adults. In Western cultures, for example, infants start sharing psychological states with others and participate in joint action with others only from 12 months of age (Liszkowski et al. 2004, 2007). Around 18 months, Western children start recognising others' goals (Warneken and Tomasello 2006) and intentions (Behne et al. 2005), and few months later, they have acquired the skills to better communicate with their social partners and engage in complex forms of cooperative behaviour (Dunfield and Kuhlmeier 2010; Brownell et al. 2009, 2013; Svetlova et al. 2010). In humans, also empathic concern for others emerges gradually (see Jensen et al. 2014). After the first 12 months, for 
instance, infants start differentiating others' distress from their own (Hoffman 2000), and from their second year of life, they start experiencing positive emotions when providing benefits to others (Aknin et al. 2012). As stressed above, despite its strong genetic component (Ebstein et al. 2010), sociality also develops throughout human lifespan as a response to the influence of the experiences and environmental stimuli received (see Maccoby and Martin 1983). These experiences may therefore have a critical effect on the development of children sociality, with long-lasting consequences throughout their lives (Bornstein et al. 2013; Lansford 2019). Findings clearly show that the development of sociality in humans is a slow and at least partly flexible process, which may be strongly affected by different environmental factors. However, whether social development in non-human primates is protracted to a similar extent as in humans is still unclear.

Comparatively little is known about how sociality develops in non-human primates, and in human cultures other than Western ones. Both inter-specific and inter-cultural approaches are however important to shed light into the evolutionary origins of human social development. This is particularly important to understand how developmental trajectories are spread across species and cultures, how ecological, demographic and social factors might alter these trajectories, and to ultimately understand what is typical in social development across human cultures.

To date, few longitudinal studies in free-ranging populations have systematically addressed how sociality develops in non-human primates (e.g. Lonsdorf et al. 2014; Kulik et al. 2015a, 2015b). Consequently, we still know little about influencing factors or circumstances encountered during early development which may affect primate sociality and hence their survival and fitness. There is evidence, for example, that patterns of social relationships differ between adult males and females, and these differences appear to emerge long before adulthood (Cords et al. 2010; Nakamichi 1989; van Noordwijk et al. 1993; Nikolei and Borries 1997). The dispersing sex, for example, tends to form looser social relationships already in the first years of life (Stumpf et al. 2009), while young philopatric females form stronger social bonds than their conspecific males (Eaton et al. 1986; Nakamichi 1989; Cords et al. 2010). Recent findings suggest that sex differences in social development do not simply result from the availability of close relatives, but follow different developmental trajectories for the two sexes (Widdig et al. 2016). Through their life, therefore, also non-human primates flexibly adapt their social behaviour, slowly growing into the social roles typical of their adult lives (Kulik et al. 2015a).

Similarly, few studies have specifically addressed whether sociality follows different developmental trajectories across human cultures. This is especially limiting, as humans are known to show important cross-cultural differences in terms of sociality, from social learning to cooperation and other forms of prosocial behaviour (see Henrich et al. 2010;
Nielsen and Haun 2015). Therefore, it is essential to understand when these inter-cultural differences emerge during development, how cultural differences affect the development of sociality and which aspects of sociality are really widespread within our species. Parenting styles, for instance, significantly vary across cultures, affecting children socio-emotional development (see Maccoby and Martin 1983; Keller 2001, 2012; Lansford 2019). The relevance of early experiences and cultural milieu in shaping human sociality suggests that developmental trajectories may crucially vary across cultures other than Western ones, with important implications in evolutionary terms (Keller 2001, 2012).

\section{Contributions to the Topical Collection}

This Topical Collection therefore aims to provide readers with an overview of the knowledge we have so far cumulated on non-human primates and across human cultures. In particular, the contributions discuss the factors that may play a major role in the development of primate sociality, and explore the impressive variability in social development across primate species and human cultures. This Topical Collection is structured in four different sections.

In the first section, we address how the development of sociality in non-human primates is affected by main bonding partners, including mothers, siblings and peers. Oliver Schülke, Julia Ostner and Andreas Berghänel open the section with a review on the effects of prenatal maternal stress on offspring social behaviour (Schülke et al. 2019). After highlighting the plasticity of social behaviour, the authors discuss the adaptiveness of such plasticity from a novel perspective, using a theoretical framework closer to behavioural ecology rather than human medical research. In particular, they discuss how prenatal stress in species with slow life histories may significantly affect offspring social behaviour and cognition in the short and long term. In his contribution, Dario Maestripieri reviews how early interactions with mothers may affect social development in non-human primates, depending on maternal characteristics such as age, rank and personality (Maestripieri 2018). He specifically discusses how mothers influence the development of species- and sextypical aspects of social behaviour, and how different maternal styles may have a long-term effect on offspring social development, including social preferences, affiliative and aggressive behaviour. In their research article, Elizabeth Lonsdorf, Margaret Stanton and Carson Murray further discuss the relevance of older siblings for infants' social development in wild chimpanzees (Lonsdorf et al. 2018). In particular, they explain how siblings form close social bonds during the first years of life and present unique long-term data to highlight important differences in social behaviour depending on the older siblings' sex. Crucially, older sisters interact more with infants 
than older brothers, affecting the social development of their younger siblings. Age peers also play a quite important role in the development of primate sociality. In her review, Elisabetta Palagi discusses how social play posits new challenges to young individuals, constituting a real springboard for social competence during the first years of life in non-human primates (Palagi 2018). In particular, she describes how social play develops, from the first interactions with mothers, to the most sophisticated rough-and-tumble sessions with peers, which allow juveniles to acquire social competence, enlarge their social networks and test their physical power and motor skills. Finally, Andrew Whiten and Erica van de Waal contribute a first review on primate social learning from a developmental perspective (Whiten and van de Waal 2018). After discussing how new experimental approaches have revealed a much wider occurrence of social learning than previously thought, already very early in development, the authors suggest that social learning can be divided into three main phases, in which the major learning models are respectively mothers, other group members and new group members after having migrated to a different group.

In the second section, we address the influence of other factors on the development of sociality in non-human primates, such as sex differences, genetic constraints and personality. In their review, Federica Amici, Lars Kulik, Doreen Langos and Anja Widdig describe how primate sociality follows different developmental trajectories depending on the infants' sex (Amici et al. 2019). In particular, they review macaques' interaction patterns with different group members throughout development, and conclude that sex differences in social behaviour emerge early on in development and increase through time. Furthermore, Christoph von Borell, Alexander Weiss and Lars Penke contribute to this section by discussing the genetic constraints of personality on the plasticity of primate behavioural development (von Borell et al. 2019). They review the mechanisms that drive the interplay of behavioural stability and flexibility, and discuss how genetic and environmental factors interact in shaping the development of primate social behaviour.

In the third section, we analyse how social cognition and multimodal communication develop through early life in nonhuman primates. In their research article, Alexandra Rosati, Alyssa Arre, Michael Platt and Laurie Santos investigate whether non-human primates, similarly to humans, show a shift in socio-emotional processing through age, based on the visual cues presented (Rosati et al. 2018). The authors report that, in contrast to humans, older rhesus macaques preferentially attend to negative stimuli, and discuss how these different ontogenetic shifts in socio-emotional processing may have important implications for more broadly understanding the evolutionary origins of social cognition. In the following contribution, Guillaume Dezecache, Catherine Crockford and Klaus Zuberbuehler investigate the development of alarm behaviour in wild chimpanzees (Dezecache et al. 2019). The authors discuss the cognitive aspects linked to alarm behaviour, including multimodal communication such as alarm calling and gaze alternation. They show that, while gaze alternation emerges early in chimpanzee development, alarm calling follows a different developmental trajectory, possibly being linked to the further emergence of more complex social cognitive skills. Furthermore, Margarita Briseno-Jaramillo, Gabriel RamosFernández, Tania Margarita Palacios-Romo, José Roberto Sosa-López and Alban Lemasson investigate spider monkeys' vocal development (Briseno-Jaramillo et al. 2018). The authors suggest that, even though the vocal repertoire of nonhuman primates is considered to have little plasticity, spider monkeys show a certain degree of flexibility when using these calls. By comparing vocalisations in adults and juveniles, in particular, they suggest that call use significantly changes through ontogeny. Finally, Marlen Fröhlich and Catherine Hobaiter review existing studies on the development of gestural communication in great apes (Fröhlich and Hobaiter 2018). While highlighting the great flexibility in their use of gestural signals, the authors discuss different possible routes of gesture acquisition, including ontogenetic ritualisation, genetic endowment, imitation and social negotiation. They conclude that, although apes' gestural repertoire appears to be largely innate, the use of gestural signals is substantially affected by individual experiences throughout ontogeny.

In the fourth and final section, we analyse how human sociality develops through ontogeny, with a special focus on cross-cultural differences in social development. The section opens with a research article by Gilda Morelli, Paula Ivey Henry and Bryn Spielvogel on food sharing in children among rural Efe foragers and Lese farmers in Congo (Morelli et al. 2019). The authors examine social and relational experiences associated with the emergence of prosocial behaviour in young children, and discuss how these experiences are culturally organised, ecologically situated and environmentally sensitive. In particular, the authors describe shared care as a form of cooperation providing children with relational opportunities crucial for their future social life, and cooperative food sharing as a process which is learnt by children through development. Interesting inter-cultural differences between the study groups also regard the role played by mothers, whose role is more central in Lese than Efe children. Furthermore, Joy Cui, Elizabeth Mistur, Christine Wei, Jennifer Lansford, Diane Putnick and Marc Bornstein contribute a detailed review on the cultural and social factors affecting early socio-emotional development across human cultures (Cui et al. 2018). In particular, the authors examine how parent-child interactions, parental discipline and parental rejection may influence children's cognitive development and behavioural adjustment across cultures. They show that positive interactions with parents are associated with the integrity of brain development, while parental rejection has a negative effect on children across 
cultures and developmental stages. Finally, Rahul Bhui, Maciej Chudek and Joseph Henrich propose a theoretical model to explain the emergence of one of the most complex forms of human social behaviour - cooperation (Bhui et al. 2019). In their paper, the authors explore the evolutionary and developmental origins of cooperation, showing that negative indirect reciprocity may suppress exploitation and create harmonious communities, favouring the adherence to community norms and the emergence of cooperation, even with minimal cognitive prerequisites.

\section{Future directions}

By bringing together comparative and cross-cultural psychologists, behavioural ecologists, field primatologists, developmental psychologists and anthropologists with different backgrounds, we provided a strong inter-disciplinary perspective on the evolutionary origins of primate social development. In this way, we hope to have drawn attention to the need for more longitudinal and comparative studies with an interdisciplinary approach. In particular, we hope that this Topical Collection will foster more studies (i) covering the entire developmental period of a given species, (ii) using a comparative perspective to better investigate differences and similarities in the social development of different species, (iii) using a more complex study design, combining behavioural observations and experimental approaches, (iv) incorporating demographical, ecological, physiological, genetic and fitness data across different life stages to determine factors or circumstances experienced during early development which affect the development of sociality and (v) combining crosscultural and developmental perspectives, to understand when inter-cultural differences emerge during development, how cultural differences affect the development of sociality and which aspects of sociality are really widespread within our species. Such an ambitious agenda can only be implemented through huge collaborative efforts. Initiatives like the ManyBabies Project (https://manybabies.github.io/) are in this respect especially promising, by setting high scientific standards and allowing the inclusion of larger sample sizes. In this way, statistical power is significantly increased, reducing the problem of low replicability that plagues research (e.g. Open Science Collaboration 2015).

This comprehensive approach will be particularly important to understand how different developmental trajectories are spread across species and cultures, and how ecological, demographic and social factors might alter these trajectories. In this way, we will not only be able to understand what is unique and typical in human social development, as compared with other taxa, but also appreciate the incredible variability in social development across primate species and human cultures.
Acknowledgements We thank the chief editors, Theo Bakker and James Traniello, for their support to the Topical Collection 'An evolutionary perspective on the development of primate sociality'. Furthermore, we would like to thank all authors and reviewers of the papers belonging to this Topical Collection for providing their expertise.

\section{Compliance with ethical standards}

Conflict of interest The authors declare they have no conflict of interest.

\section{References}

Aknin LB, Hamlin JK, Dunn EW (2012) Giving leads to happiness in young children. PLoS One 7:e39211

Amici F, Kulik L, Langos D, Widdig A (2019) Growing into adulthooda review on sex differences in the development of sociality across macaques. Behav Ecol Sociobiol 73:18

Archie EA, Tung J, Clark M, Altmann J, Alberts SC (2014) Social affiliation matters: both same-sex and opposite-sex relationships predict survival in wild female baboons. Proc R Soc B 281:20141261

Behne T, Carpenter M, Call J, Tomasello M (2005) Unwilling versus unable: infants' understanding of intentional action. Dev Psychol 41:328-337

Bhui R, Chudek M, Henrich, J (2019) How exploitation launched human cooperation. Behav Ecol Sociobiol 73:78

Bornstein MH, Hahn CS, Suwalsky JTD (2013) Developmental pathways among adaptive functioning and externalizing and internalizing behavioral problems: cascades from childhood into adolescence. Appl Dev Sci 17:76-87

Briseno-Jaramillo M, Ramos-Fernández G, Palacios-Romo TM, SosaLópez JR, Lemasson A (2018) Age and social affinity effects on contact call interactions in free-ranging spider monkeys. Behav Ecol Sociobiol 72:192

Brownell CA, Svetlova M, Nichols S (2009) To share or not to share: when do toddlers respond to another's needs? Infancy 14:117-130

Brownell CA, Iesue SS, Nichols SR, Svetlova M (2013) Mine or yours? Development of sharing in toddlers in relation to ownership understanding. Child Dev 84:906-920

Chapais B (2001) Primate nepotism: what is the explanatory value of kin selection? Int J Primatol 22:203-229

Cords M, Sheehan MJ, Ekernas LS (2010) Sex and age differences in juvenile social priorities in female philopatric, nondespotic blue monkeys. Am J Primatol 72:193-205

Crockford C, Wittig RM, Whitten PL, Seyfarth RM, Cheney DL (2008) Social stressors and coping mechanisms in wild female baboons (Papio hamadryas ursinus). Horm Behav 53:254-265

Cui J, Mistur EJ, Wei C, Lansford JE, Putnick DL, Bornstein MH (2018) Multilevel factors affecting early socioemotional development in humans. Behav Ecol Sociobiol 72:172

Dezecache G, Crockford C, Zuberbuehler K (2019) The development of communication in alarm contexts in wild chimpanzees Behav Ecol Sociobiol in press

Dunfield KA, Kuhlmeier VA (2010) Intention-mediated selective helping in infancy. Psychol Sci 21:523-527

Eaton GG, Johnson DF, Glick BB, Worlein JM (1986) Japanese macaques (Macaca fuscata) social development: sex differences in juvenile behavior. Primates 27:141-150

Ebstein RP, Israel S, Chew SH, Zhong S, Knafo A (2010) Genetics of human social behavior. Neuron $65: 831-844$

Engh AL, Beehner JC, Bergman TJ, Whitten PL, Hoffmeier RR, Seyfarth RM, Cheney DL (2006a) Behavioural and hormonal responses to predation in female chacma baboons (Papio hamadryas ursinus). Proc R Soc Lond B 273:707-712 
Engh AL, Beehner JC, Bergman TJ, Whitten PL, Hoffmeier RR, Seyfarth RM, Cheney DL (2006b) Female hierarchy instability, male immigration and infanticide increase glucocorticoid levels in female chacma baboons. Anim Behav 71:1227-1237

Fröhlich M, Hobaiter C (2018) The development of gestural communication in great apes. Behav Ecol Sociobiol 72:194

Gilby IC, Brent LJ, Wroblewski EE, Rudicell RS, Hahn BH, Goodall J, Pusey AE (2013) Fitness benefits of coalitionary aggression in male chimpanzees. Behav Ecol Sociobiol 67:373-381

Haunhorst CB, Heesen M, Ostner J, Schülke O (2017) Social bonds with males lower the costs of competition for wild female Assamese macaques. Anim Behav 125:51-60

Henrich J, Heine SJ, Norenzayan A (2010) The weirdest people in the world? Behav Brain Sci 33:61-135

Hoffman ML (2000) Empathy and moral development: implications for caring and justice. Cambridge University Press, New York

Holt-Lunstad J, Smith TB, Layton JB (2010) Social relationships and mortality risk: a meta-analytic review. PLoS Med 7:e1000316

Jensen K, Vaish A, Schmidt MFH (2014) The emergence of human prosociality: aligning with others through feelings, concerns, and norms. Front Psychol 5:822

Keller H (2001) Lifespan development: evolutionary perspectives. In: Smelser NJ, Baltes PB (eds) International encyclopedia of the social and behavioral sciences. Elsevier Science, Oxford, pp 8840-8844

Keller H (2012) Cross-cultural psychology: taking people, contexts, and situations seriously. In: Valsiner J (ed) The Oxford handbook of culture and psychology. Oxford University Press, Oxford, pp 116131

King AJ, Clark FE, Cowlishaw G (2012) The dining etiquette of desert baboons: the roles of social bonds, kinship, and dominance in cofeeding networks. Am J Primatol 73:768-774

Kulik L, Muniz L, Mundry R, Widdig A (2012) Patterns of interventions and the effect of coalitions and sociality on male fitness. Mol Ecol 21:699-714

Kulik L, Amici F, Langos D, Widdig A (2015a) Sex differences in the development of social relationships in rhesus macaques (Macaca mulatta). Int J Primatol 36:353-376

Kulik L, Amici F, Langos D, Widdig A (2015b) Sex differences in the development of aggressive behavior in rhesus macaques (Macaca mulatta). Int J Primatol 36:764-789

Lansford JE (2019) Parenting and child discipline. In: Bornstein MH (ed) Handbook of parenting, vol vol. 3. Routledge, New York, pp 65-90

Liszkowski U, Carpenter M, Henning A, Striano T, Tomasello M (2004) Twelve-montholds point to share attention and interest. Dev Sci 7: 297-307

Liszkowski U, Carpenter M, Tomasello M (2007) Reference and attitude in infant pointing. J Child Lang 34:1-20

Lonsdorf EV, Markham AC, Heintz MR, Anderson KE, Ciuk DJ, Goodall J, Murray CM (2014) Sex differences in wild chimpanzee behavior emerge during infancy. PLoS One 9:e99099

Lonsdorf EV, Stanton MA, Murray CM (2018) Sex differences in maternal sibling-infant interactions in wild chimpanzees. Behav Ecol Sociobiol 72:117

Maccoby EE, Martin JA (1983) Socialization in the context of the family: parent-child interaction. In: Mussen PH, Hetherington EM (eds) In Handbook of child psychology: socialization, personality, and social development. Wiley, New York, pp 1-101

Maestripieri D (2018) Maternal influences on primate social development. Behav Ecol Sociobiol 72:130

Michelleta J, Waller BM, Panggur MR, Neumann C, Duboscq J, Agil M, Engelhardt A (2012) Social bonds affect anti-predator behaviour in a tolerant species of macaque, Macaca nigra. Proc R Soc Lond B 279: $4042-4050$

Morelli G, Henry PI, Spielvogel B (2019) Learning prosociality: insights from young forager and subsistence farmer children's food sharing with mothers and others. Behav Ecol Sociobiol 73:86
Moscovice LR, Heesen M, Di Fiore A, Seyfarth RM, Cheney DL (2009) Paternity alone does not predict long-term investment in juveniles by male baboons. Behav Ecol Sociobiol 63:1471-1482

Nakamichi M (1989) Sex differences in social development during the first 4 years in a free-ranging group of Japanese monkeys, Macaca fuscata. Anim Behav 38:737-748

Nguyen N, Van Horn RC, Alberts SC, Altmann J (2009) "Friendships" between new mothers and adult males: adaptive benefits and determinants in wild baboons (Papio cynocephalus). Behav Ecol Sociobiol 63:1331-1344

Nielsen M, Haun DBM (2015) Why developmental psychology is incomplete without comparative and cross-cultural perspectives. Philos Trans R Soc B 371:20150071

Nikolei J, Borries C (1997) Sex differential behavior of immature hanuman langurs (Presbytis entellus) in Ramnagar, South Nepal. Int J Primatol 18:415-437

Open Science Collaboration (2015) Estimating the reproducibility of psychological science. Science 349:6251

Palagi E (2018) Not just for fun! Social play as a springboard for adult social competence in human and non-human primates. Behav Ecol Sociobiol 72:90

Rosati AG, Arre AM, Platt ML, Santos LR (2018) Developmental shifts in social cognition: socio-emotional biases across the lifespan in rhesus monkeys. Behav Ecol Sociobiol 72:163

Schülke O, Bhagavatula J, Vigilant L, Ostner J (2010) Social bonds enhance reproductive success in male macaques. Curr Biol 20: $2207-2210$

Schülke O, Ostner J, Berghänel A (2019) Prenatal maternal stress effects on the development of primate social behavior. Behav Ecol Sociobiol in press

Seyfarth RM, Cheney DL (2012) The evolutionary origins of friendship. Annu Rev Psychol 63:153-177

Silk JB (2002a) Kin selection in primate groups. Int J Primatol 23:849_ 875

Silk JB (2002b) Using the 'F'-word in primatology. Behaviour 139:421446

Silk JB (2014) Evolutionary perspectives on the links between close social bonds, health, and fitness. In: Weinstein M, Lane MA (eds) Sociality, hierarchy, health: comparative biodemography: a collection of papers. National Academies Press, Washington, pp 121-144

Silk JB, Alberts SC, Altmann J (2003) Social bonds of female baboons enhance infant survival. Science 302:1231-1234

Silk JB, Beehner JC, Bergman TJ, Crockford C, Engh AL, Moscovice LR, Wittig RM, Seyfarth RM, Cheney DL (2009) The benefits of social capital: close social bonds among female baboons enhance offspring survival. Proc R Soc Lond B 276:3099-3104

Silk JB, Beehner JC, Bergman TJ, Crockford C, Engh AL, Moscovice LR, Wittig RM, Seyfarth RM, Cheney DL (2010) Strong and consistent social bonds enhance the longevity of female baboons. Curr Biol 20:1359-1361

Smith KP, Christakis NA (2008) Social networks and health. Annu Rev Sociol 34:405-429

Stumpf RM, Emery Thompson M, Muller MN, Wrangham RW (2009) The context of female dispersal in Kanyawara chimpanzees. Behaviour 146:629-656

Svetlova M, Nichols SR, Brownell CA (2010) Toddlers' prosocial behavior: from instrumental to empathic to altruistic helping. Child Dev 81:1814-1827

Thompson NA, Cords M (2018) Stronger social bonds do not always predict greater longevity in a gregarious primate. Ecol Evol 8: 1604-1614

Tung J, Archie EA, Altmann J, Alberts SC (2016) Cumulative early life adversity predicts longevity in wild baboons. Nat Commun 7:11181

van Noordwijk MA, Hemelrijk CK, Herremans LAM, Sterck EHM (1993) Spatial position and behavioral sex differences in juvenile long-tailed macaques. In: Pereira ME, Fairbanks LA (eds) Juvenile 
primates: life history, development and behavior. University of Chicago Press, Chicago, pp 77-85

von Borell CJ, Weiss A, Penke L (2019) Developing individual differences in primate behavior: the role of genes, environment, and their interplay. Behav Ecol Sociobiol 73:20

Warneken F, Tomasello M (2006) Altruistic helping in human infants and young chimpanzees. Science 311:1301-1303

Whiten A, van de Waal E (2018) The pervasive role of social learning in primate lifetime development. Behav Ecol Sociobiol 72:80

Widdig A, Langos D, Kulik L (2016) Sex differences in kin bias at maturation: male rhesus macaques prefer paternal kin prior to natal dispersal. Am J Primatol 78:78-91
Wingfield JC, Sapolsky RM (2003) Reproduction and resistance to stress: when and how. J Neuroendocrinol 15:711-724

Wittig RM, Crockford C, Lehmann J, Whitten PL, Seyfarth RM, Cheney DL (2008) Focused grooming networks and stress alleviation in wild female baboons. Horm Behav 54:170-177

Wittig RM, Crockford C, Weltring A, Langergraber KE, Deschner T, Zuberbühler K (2016) Social support reduces stress hormone levels in wild chimpanzees across stressful events and everyday affiliations. Nat Commun 7:13361

Publisher's note Springer Nature remains neutral with regard to jurisdictional claims in published maps and institutional affiliations. 